\title{
Transformation of Citizenship and Governance in Asia: The Challenges of Social and Mobile Media
}

\author{
Nojin Kwak*, Ines Mergel ${ }^{* *}$, Peter Parycek***, Marko Skoric ${ }^{* * * *}$ \\ *University of Michigan, USA; kwak@umich.edu \\ ** Maxwell School of Citizenship and Public Affairs, Syracuse University; iamergel@maxwell.syr.edu \\ *** Danube University Krems; peter.parycek@donau-uni.ac.at \\ **** City University of Hong Kong; Mskoric@cityu.edu.hk
}

\begin{abstract}
The eJournal of eDemocracy and Open Government (JeDEM) is a peer-reviewed, Open Access journal (ISSN: 2075-9517) published twice a year. It addresses theory and practice in the areas of eDemocracy and Open Government as well as eGovernment, eParticipation, and eSociety. JeDEM publishes ongoing and completed research, case studies and project descriptions that are selected after a rigorous blind review by experts in the field.
\end{abstract}

More information: www.jedem.org

Keywords: Asia, Citizenship, Governance, Social Media, Mobile Media

$\mathrm{T}$ he proliferation of social media and mobile phones over the last decade has spurred significant interest in their civic and political implications not only within the scholarly community, but also among journalists, practitioners, activists, policy-makers, and ordinary citizens. The emerging social and mobile media-enabled practices, including content generation, self-expression, collaboration, and network organization, are changing our understanding of civic action, good governance and politics in general.

The decreasing cost of participation, paired with an improved speed and efficiency of information dissemination, mobilization and organization have made social media an indispensible means for civic and political action. The new platforms have enabled citizens to interact with governments and other political institutions more directly, monitor their functioning, and take more active role in the policy-making processes. While the above changes are already widely discussed in mature, developed Western democracies, there is an even greater need to address them in the context of rapidly developing Asian societies. Although countries in Asia vary greatly in terms of the levels of economic and political development, quality of information and communication infrastructure, as well as their cultural, political and religious traditions, the arrival of the Internet and social media has led to some similar socio-political shifts. Those include an increasing diversity of voices in the public sphere, greater visibility of political discourse, increased demands for transparency and accountability, and a significantly improved capacity for decentralized civic and political action.

This special issue showcases innovative scholarly works examining the role of social and mobile media in the formation and/or transformation of democratic citizenship in some of the most populous and economically powerful countries in Asia-China, India, Japan and South Korea. Although methodologically and thematically diverse, the four studies included here all demonstrate an important, evolving role of social media platforms in facilitating citizen expression and action. The findings indicate that social media use is associated with certain democratic benefits, including amplifying citizen calls for greater government accountability and transparency (Pang), exposing citizens to diverse political perspectives (Bae), and facilitating rapid communication and efficient 
coordination during civic protests (Jaidka). Still, our contributors also point out to the less desirable outcomes brought by social media platforms such as corporate-sponsored spread of malicious rumors and falsehoods in China (Pang) and reporting and dissemination of inflammatory, onesided and xenophobic news by anonymous online activists in Japan (Kaigo).

The special issues opens with Pang's analysis of the impact of social media in the most populous country in the world-China. Although mainstream news reports choose to focus solely on the empowerment of Chinese citizens via social media, Pang's study shows both the bright and the dark sides of social media in China. The author examines and compares two indicidents that illustrate the power of social media: the aftermath of a train collision in Wenzhou in 2011 and an elaborate smear campaign organized by a Chinese dairy company to take out its competition. While the former case shows that social media can significantly amplify citizens' calls for greater accountability and transparency in an authoritarian state, the latter demonstrates how social networks can be strategically utilized by commercial interests to spread malicious rumors and misinform the public. Pang's study suggests that because social media networks are typically imbued with trust and credibility, they are increasingly playing important informational and mobilizational roles in the public life in China. However, social media use may not always lead to democratically desirable effects, as the misuses and abuses of these platforms are also likely to happen, particularly given low perceived credibility of Chinese mainstream media sources.

Soo Young Bae's study, titled "A Trigger or a Muffler?," examined the role of social media in the 2012 presidential election in S. Korea. Utilizing a national data set, collected via an online survey, Bae attempts to address a series of questions concerning S. Korean voters' encounters with political opinions that challenge their own viewpoints (i.e., crosscutting exposure) on social networking sites (SNS). In particular, Bae inquires about the possibility that social media users are more likely to encounter dissonant views online, some of which could be accidental, and that therefore social media may essentially hinder one's willingness to express their views. While this line of inquiry that examines the impact of crosscutting exposure is consistent with prior research in political talk (and thus not new), Bae successfully complicates the inquiry by factoring into its theoretical model one of the core characteristics of the new media environment, "socialness" or "online friendship." Findings of the study suggest that cross-cutting exposure in the $2012 \mathrm{~S}$. Korean presidential election fostered political expression of SNS users, particularly younger users, when social media users believed that their online friends were supportive of their political views. ${ }^{1}$

The role of social media in facilitating and coordinating protests and social movements has gained attention in research, in particular since the Arab Spring, which was even referred to as "Twitter Revolution". Jaidka's study offers a comprehensive approach towards analysing the role of this medium by analysing themes, usage characterstistics, and users on Twitter concerninga public outcry against a gangrape incident in Delhi (\#delhigangrape). The study examines the role of Twitter users as citizen journalists, the role of the medium for shifting the balance of media powers, and major role players on Twitter during the days of the protest. Results suggest that Twitter as an information sharing network is disengaging police control over information. However, findings also reaffirm that traditional media still play a critical role in disseminating information during protests.

Kaigo's study provides insights into how the Japanese information aggregation online site, Matome Saito, compiles and summarizes political online messages posted on the Ni-Channel surrounding the 2012 election. A content analysis of 1,750 blog pages leading up to the election shows how the aggregation mechanisms led to more favorable language toward the Liberal Democratic Party in Japan in comparison to other parties. Especially the far-right wing Japan Restoration Party was negatively represented on the message aggregation site. With this study Kaigo shows how an extremely popular information dissemination system that is widely adopted by Japanese youth can influence political opinions.

1 This work was supported by the Academy of Korean Studies (Korean Studies Promotion Service) Grant funded by the Government of the Republic of Korea (Ministry of Education) (AKS-2011-BAA-2102). 
In addition to the special section focused on Asia, this issue of JeDEM also features peer-reviewed research papers in the field of eDemocracy and Open Government.

There is a broad consensus that transparency is a core value of democracy, and many studies investigate to what extent the new new information and communication technologies have a positive impact on governance cultures, for instance a culture of transparency in the public sector. Contributing to this field of research, Vieira's study aims to assess the association between egovernment quality and the occurrence of corruption in Brazil. The paper is an attempt to shed light on the causes of corruption through empirical analysis of the impact of public transparency mechanisms on the frequency of improbities in Brazilian municipalities. Results indicate that egovernment mechanisms are negatively associated with improbities in Brazilian local government, as e-Government initiatives are perceived to increase the costs of corrupt transactions and to reduce the benefits of engaging in corrupt practices.

In summary, this issue of JeDEM hopefully demonstrates both the significance as well as complexity of the transformations of citizenship and governance happening in the socia media era. By offering our readers a glimpse of cutting-edge research conducted outside the United States and Europe we hope to inspire new approaches for studying the above phenomena, and encourage policy-makers, activists, and practitioners to harness the potential of emerging media platforms for the common good.

\section{About the Authors}

Nojin Kwak (Ph.D., University of Wisconsin-Madison) is an associate professor in the Department of Communication Studies and Director of the Nam Center for Korean Studies at the University of Michigan. His recent studies analyze the impact of internet and mobile communication technologies on community involvement, deliberative openness, and political participation. Kwak has received numerous top paper awards at major conferences. Kwak is a co-editor of a book series, Perspectives on Contemporary Korea, published by the University of Michigan Press.

Dr. Ines Mergel is Assistant Professor of Public Administration and International Affairs at Syracuse University's Maxwell School of Citizenship and Public Affairs, Syracuse, NY. Her research and teaching focuses on the diffusion and adoption of new technologies in the public sector and specifically on the professional use of social media applications to increase transparency, participation and collaboration in the U.S. federal government.

Peter Parycek, PhD, MSc, is Head of the Center for E-Governance at the Danube University Krems and Chairman of the ministerial working groups "E-Democracy \& E-participation" and "E-Government Training" at the Austrian Federal Chancellery. As a lawyer and graduate of the Master's program Telematics, his work is at the intersection of legal policy, social and technological developments. His research and project priorities include eGovernance, eDemocracy and eGovernment. He is responsible for the conference series CeDEM (International Conference for e-Democracy and Open Government) and the open access journal JeDEM (eJournal of eDemocracy and Open Government).

Marko M. Skoric is Associate Professor at the Department of Media and Communication, City University of Hong Kong. Previously, he was Assistant Professor at the Wee Kim Wee School of Communication and Information, Nanyang Technological University, Singapore. He holds a Ph.D. in Communication from the University of Michigan, and a B.Sc. in Psychology from the University College London, UK. Marko's teaching and research interests are focused on new media and social change, with particular emphasis on civic and political implications of new communication technologies. 